\title{
Pinning control of cluster synchronization in regular networks
}

\author{
Liang Wang, Yali Guo, Ya Wang, Huawei Fan, and Xingang Wang ๑* \\ School of Physics and Information Technology, Shaanxi Normal University, Xi'an 710062, China
}

(Received 7 January 2020; accepted 30 March 2020; published 27 April 2020)

\begin{abstract}
As an efficient approach to controlling the collective dynamics in networked systems, pinning synchronization has been extensively studied in the literature. Different from the existing studies in which the target is normally chosen as the global synchronization state, here we investigate the pinning control of cluster synchronization in regular networks, including the globally connected and ring-structure networks. For globally connected networks, it is found that when a fraction of nodes is pinned, the group of pinning nodes will form a synchronous cluster, while the unpinning nodes are always in the desynchronization state. We conduct a theoretical analysis of the stability of the synchronous cluster, which shows that the critical pinning strength for controlling cluster is inversely proportionally to the uniform coupling strength, but is independent of the number of pinning nodes. For ring-structure networks, however, it is found that the properties of the synchronous clusters, including the sizes and partition of the clusters, are affected by both the number of pinning nodes and the pinning locations. Theoretical analysis on the stabilities of the clusters shows that different from globally connected networks, the introduction of pinnings to the ring-structure network will degenerate the network symmetry, which in turn determines the properties of the clusters.
\end{abstract}

DOI: 10.1103/PhysRevResearch.2.023084

\section{INTRODUCTION}

Cluster synchronization (CS), also known as partial or group synchronization, refers to the coherent motion of partial oscillators in large-size systems [1], which, for its significant implications to the functionalities of many natural and manmade systems, has been extensively studied by researchers from different fields over the past two decades [2-10]. Different from global synchronization in which all oscillators are synchronized to the same trajectory, in CS the oscillators are synchronized to different trajectories. Specifically, the oscillators are organized into different groups (clusters), with oscillators within the same group being synchronized, but are not for oscillators from different groups. For systems with regular coupling structures, e.g., the lattices, CS is generated normally by the mechanism of symmetry breaking [11-13], and the spatial property of the synchronization pattern can be analyzed by methods such as the master stability function (MSF) [14-16]. Recently, stimulated by the rapid progress of network science $[17,18]$, the generation of CS on networks of complex coupling structures has attracted much attention [19-32]. In exploring the generation of CS in complex networks, two of the key questions that are of broad interest are (1) how to identify, from the network structure, the possible CS states and (2) how to analyze the stabilities of these

\footnotetext{
*wangxg@snnu.edu.cn

Published by the American Physical Society under the terms of the Creative Commons Attribution 4.0 International license. Further distribution of this work must maintain attribution to the author(s) and the published article's title, journal citation, and DOI.
}

CS states. These questions have led to the development of new techniques for node partitions, e.g., the external equitable partitions (EEPs) and symmetry-based orbit partitions $[23,29,30]$, as well as new methods of stability analysis, e.g., the symmetry-based stability analysis and Lyapunovfunction-based analysis [19,23,25,33,34].

For either regular or complex networks, a general finding in previous studies is that compared with global synchronization, the generation of CS is much more difficult [19-32]. For instance, CS is observed normally in a narrow region in the parameter space of coupling strength and is fragile to noise perturbations $[7,9,10,31]$. This is particularly the case where complex networks are concerned, in which the emergence of CS is also restricted by the network topology $[23,25,26,28-$ 30], e.g., the network permutation symmetries. Whereas it is difficult to generate CS by the self-organization of the networked nodes, CS might be generated with the help of some control techniques. Guided by this idea, efforts have been made in recent years on the control of CS in networked oscillators in which some effective control schemes have been proposed, such as adapting the weights of the network links $[31,34]$, slightly adjusting the network structure $[21,32]$, and pinning the network nodes by an external controller or a smallsize control network [25,26,33]. Among the schemes, pinning control is distinguished from others in feasibility (e.g., keeping the network unchanged), efficiency (e.g., pinning only a fraction of the nodes), and flexibility (e.g., controlling the network to different CS states), and is considered to be an important approach to the control of spatiotemporal behaviors in a variety of dynamical systems [25,26,33,35-38].

When applied to complex networks, however, the pinning scheme encounters some problems [25,26,33]. First, to control CS in a complex network, we need to identify from the 
network structure the possible CS states that are solutions of the system dynamics. While this can be done in general by techniques such as EEP and permutation symmetries [23,29], the partitions of the network nodes are unique and fixed. As the formation of the synchronous clusters relies on the node partitions, the number of CS states that the network can be controlled to thus is limited. Second, in pinning control of CS in a complex network, every node will receive a pinning signal from the controller [25,26], i.e., the network is globally pinned. The adoption of global pinning is necessary for controlling CS in complex networks because, in introducing the pinnings, one needs to keep the node partitions unchanged [33]. Otherwise, if the network nodes are partially pinned, it is highly possible that the node partitions will be different from the original partitions. In such a case, the desired CS state will no longer be a solution of the system dynamics, making the control failed. For these concerns, the pinning control of CS in complex networks remains a challenging problem.

Instead of complex networks, in the present work we study the pinning control of CS states in regular networks. Compared to complex networks, regular networks possess high-order symmetries which, in the presence of pinnings, could be degenerated into different low-order symmetries. In particular, by changing the number and the locations of the pinning nodes, the network symmetry can be degenerated into a variety of low-order symmetries, providing ample room for different CS states. Inspired by this, we introduce pinnings to a fraction of nodes in two types of regular networks, namely, the globally connected and ring-structure networks, and investigate the responses of the network dynamics to the change of the pinning configurations. We are able to demonstrate numerically and argue mathematically the following: (1) When a fraction of nodes in a globally connected network is pinned, the group of pinning nodes will be synchronized if the pinning strength exceeds a critical value, while the unpinning nodes are always desynchronized. The critical pinning strength is dependent on the uniform coupling strength, but is independent of the fraction of pinning nodes. (2) When a fraction of nodes in a ring-structure network is pinned, synchronization can also be achieved among the unpinning nodes, showing the phenomenon of multiple-cluster synchronization. Moreover, by changing the number of pinning nodes and the pinning locations, the same network can be controlled into different CS states. These findings enrich our understanding of the dynamical responses of complex networks to external signals, and might provide an alternative approach to the control of the nonlinear dynamics in complex systems.

\section{MODEL}

Our model of networked oscillators under pinning control reads $[25,26,35-38]$

$$
\dot{\mathbf{x}}_{i^{\prime}}=\mathbf{F}_{i^{\prime}}\left(\mathbf{x}_{i^{\prime}}\right)+\varepsilon \sum_{j^{\prime}=1}^{N^{\prime}} w_{i^{\prime} j^{\prime}} \mathbf{H}\left(\mathbf{x}_{j^{\prime}}\right)+\varepsilon \eta_{i^{\prime}}\left[\mathbf{H}\left(\mathbf{x}_{T}\right)-\mathbf{H}\left(\mathbf{x}_{i^{\prime}}\right)\right]
$$

with $i^{\prime}, j^{\prime}=1, \ldots, N^{\prime}$ the oscillator (node) indices and $N^{\prime}$ the network size. In Eq. (1), $\mathbf{x}_{i^{\prime}}$ represents the state vector of the $i^{\prime}$ th oscillator, $\mathbf{H}(\mathbf{x})$ denotes the coupling function, and $\varepsilon$ is the uniform coupling strength. In the isolated form, the dynamics of the $i^{\prime}$ th oscillator is governed by the equation $\dot{\mathbf{x}}_{i^{\prime}}=\mathbf{F}_{i^{\prime}}\left(\mathbf{x}_{i^{\prime}}\right)$, which, for the sake of simplicity, is set as identical among the nodes, i.e., $\mathbf{F}_{i^{\prime}}(\mathbf{x})=\mathbf{F}(\mathbf{x})$. The coupling relationship among the oscillators, i.e., the network structure, is encoded in the coupling matrix $\mathbf{W}=\left\{w_{i^{\prime} j^{\prime}}\right\}_{N^{\prime} \times N^{\prime}}$, with $w_{i^{\prime} j^{\prime}}=1 / k_{i^{\prime}}$ if there is a link between nodes $i^{\prime}$ and $j^{\prime}$, otherwise $w_{i^{\prime} j^{\prime}}=0$. Here, $k_{i^{\prime}}$ denotes the number of links associated with node $i^{\prime}$, i.e., the node degree. The diagonal elements are set as $w_{i^{\prime} i^{\prime}}=$ $-\sum_{j^{\prime}} w_{i^{\prime} j^{\prime}}$. As such, the row sum of the elements equals 0 and $\mathbf{W}$ is a Laplacian matrix. The state of the external controller is represented by $\mathbf{x}_{T}$, which follows the same dynamics as the isolated node. By the same function $\mathbf{H}(\mathbf{x})$, the controller is coupled unidirectionally to a fraction of nodes in the network, with the normalized pinning strength being uniformly set as $\eta$. The set of nodes receiving pinnings from the controller is represented as $V_{p}=\{l\}$, with $l=1, \ldots, m$ the index of the pinning nodes. For node $i^{\prime}$, we have $\eta_{i^{\prime}}=\eta$ if $i^{\prime} \in V_{p}$, otherwise $\eta_{i^{\prime}}=0$.

Regarding the controller as an additional node to the original network, the whole system can be treated as an enlarged network containing $N=N^{\prime}+1$ nodes. The dynamics of the enlarged network is governed by equations

$$
\dot{\mathbf{x}}_{i}=\mathbf{F}\left(\mathbf{x}_{i}\right)+\varepsilon \sum_{j=1}^{N} c_{i j} \mathbf{H}\left(\mathbf{x}_{j}\right)
$$

with $\mathbf{C}=\left\{c_{i j}\right\}_{N \times N}$ the coupling matrix of the enlarged network. The matrix $\mathbf{C}$ is constructed from $\mathbf{W}$ as follows. For $1 \leqslant i, j \leqslant N-1$ and $i \neq j$, we have $c_{i j}=w_{i j}$. For elements in the last column of $\mathbf{C}$, we set $c_{i N}=\eta$ if $i \in V_{p}$, otherwise $c_{i N}=0$. All elements in the last row of $\mathbf{C}$ are set as 0 , i.e., $c_{N, j}=0$ for $j=1, \ldots, N$. As such, the controller is indexed as the $N$ th node in the enlarged network, and the set of pinning nodes is encoded in the last column of $\mathbf{C}$. The diagonal elements of $\mathbf{C}$ are set as $c_{i i}=w_{i i}-\eta$ for $i \in V_{p}$, otherwise $c_{i i}=w_{i i}$.

The model described by Eqs. (1) and (2) have been employed widely in the literature for exploring the synchronization behaviors of networked oscillators under pinning couplings [25,26,35-38]. It is worth mentioning that in previous studies, the mission of pinning control is mainly focused on driving the whole network from the desynchronization state to the global synchronization state. In such a case, as the controller is coupled unidirectionally to a fraction of nodes in the network, the manifold of the global synchronization state is defined by the trajectory of the controller. This makes the problem of network control essentially a problem of network synchronization, and the network controllability can be analyzed by the conventional method of MSF [14-16]. This is the reason why we rewrite Eq. (1) as Eq. (2). However, when the targets are CS states, the synchronization manifolds are no longer defined by the controller, but are dependent on the network symmetries. In this case, the conventional MSF cannot be applied directly and a generalized method should be employed. As we will show later, even for CS states, we are still able to treat the problem of pinning control from the point of view of network synchronization. 


\section{NUMERICAL RESULTS}

\section{A. Pinning CS in globally connected network}

We start by investigating numerically the pinning control of CS in globally coupled networks. In simulations, we set the network size as $N^{\prime}=10$, and adopt the chaotic Lorenz oscillators as the nodal dynamics. In the isolated form, the Lorenz oscillator is described by the equations $(d x / d t, d y / d t, d z / d t)=[\sigma(y-x), x(\rho-z)-y, x y-$ $\beta z]$. The parameters of the Lorenz oscillators are set as $(\sigma, \rho, \beta)=(10,35,8 / 3)$, with which the isolated oscillators are chaotic. The coupling function is chosen as $\mathbf{H}([\mathbf{x}, \mathbf{y}, \mathbf{z}])=$ $[0, x, 0]^{T}$, i.e., the $x$ variable is coupled to the $y$ variable between the connected oscillators. The critical coupling strength for global synchronization, $\varepsilon_{c}$, can be analyzed by the standard MSF method, from which we have $\varepsilon_{c} \approx 7.5$. In our study, we set $\varepsilon=5.0$, so as to make the network stay in the desynchronization state without pinning control. In implementing the control, we randomly chose $m=5$ nodes in the network and introduced pinnings to them according to Eq. (1). (Note that due to the network symmetry, the pinning nodes can be chosen randomly while the system dynamics is keeping unchanged.)

The time evolutions of the oscillators under different pinning strengths are plotted in Fig. 1(a). We see that when the pinning strength is small $(\eta=0.3)$ [Fig. 1(a1)], there are $N=11$ distinct trajectories, indicating the absence of any synchronization between the nodes (including the controller). However, when the pinning strength is large $(\eta=$ 0.55) [Fig. 1(a2)], we see that after a transient period about $T=110$ (the length of the transient period is affected by the initial conditions and the system parameters), $m=5$ of the trajectories are converged into a single one, while the remaining trajectories (including the controller) remain as different from each other. That is, a synchronous cluster containing $m=5$ oscillators is formed. By checking the node indices, it is revealed that the group of synchronized nodes is just those receiving the pinnings from the controller. This unique CS state, which is named isolated desynchronization in Ref. [23], is analogous to the chimeralike states observed in regular networks of nonlocally coupled phase oscillators [39,40]. It is worth mentioning that different from chimeralike states which are generated by the mechanism of spontaneous symmetry breaking of the system dynamics, here the symmetry breaking is induced by the pinning couplings.

To find the critical pinning strength that generates CS, we plot in Fig. 1(b) the variation of the cluster synchronization error as a function of $\eta$. Here, cluster synchronization error is defined as $\langle\delta x\rangle_{1}=\sum_{i=1}^{m}\left|x_{i}-\bar{x}_{1}\right| / m$, with $i=1, \ldots, m$ the index of the pinning nodes and $\bar{x}_{1}=\sum_{i=1}^{m} x_{i} / m$ the averaged state of the pinning nodes. The cluster synchronization error is evaluated after a transient period of $T=1 \times 10^{3}$. We see that $\langle\delta x\rangle_{1}$ is decreased to 0 at about $\eta_{c}=0.5$. (In the present work, the value of $\eta_{c}$ is defined as the point where the cluster synchronization error is smaller than $10^{-3}$ ). Figure $1(\mathrm{~b})$ also shows the synchronization error of the unpinning nodes, $\langle\delta x\rangle_{2}=\sum_{j=m+1}^{N-1}\left|x_{j}-\bar{x}_{2}\right| /(N-m-1)$ (here, $\bar{x}_{2}$ is the averaged state of the unpinning nodes), and the synchronization error between the controller and the averaged state of the pinning nodes, $\delta x_{T}=\left|x_{T}-\bar{x}_{1}\right|$. We see that both $\langle\delta x\rangle_{2}$ and $\delta x_{T}$ remain at large values at $\eta_{c}$, indicating that (1) the
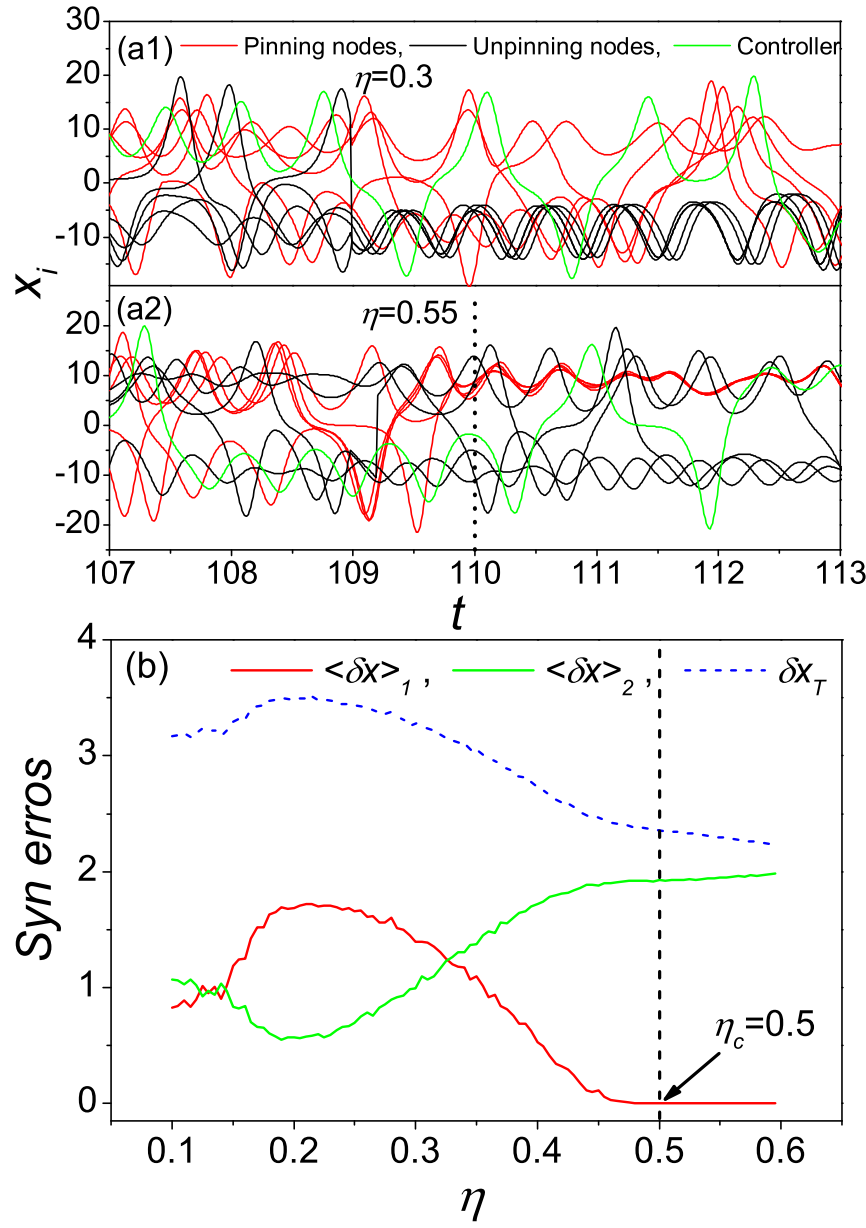

FIG. 1. Pinning control of CS in globally connected network. The network contains $N^{\prime}=10$ nodes, and pinnings are added on $m=5$ nodes. (a1),(a2) For $\varepsilon=5.0$, the time evolution of the node states under different pinning strengths: (a1) $\eta=0.3$ and (a2) $\eta=$ 0.55 . The evolutions of the pinning and unpinning nodes are colored in red and black, respectively. The evolution of the controller is colored in green. In (a2), the trajectories of the pinning nodes are converged to a single one after a transient period about $T=110$. (b) The variations of different synchronization errors with respect to the pinning strength. $\langle\delta x\rangle_{1}$ denotes the synchronization error among the pinning nodes, which reaches 0 at about $\eta_{c}=0.5 .\langle\delta x\rangle_{2}$ denotes the synchronization error among the unpinning nodes. $\delta x_{T}$ is the synchronization error between the averaged state of the pinning nodes and the controller. See the text for the detailed definitions of the synchronization errors.

unpinning nodes are not synchronized and (2) the pinning nodes are synchronized to a trajectory that is different from that of the controller.

Whether the critical pinning strength is dependent on the uniform coupling strength? To check out, we plot in Fig. 2(a) the variation of $\eta_{c}$ with respect to $\varepsilon$. We see that with the increase of $\varepsilon$, the value of $\eta_{c}$ is decreased monotonically. This phenomenon is expected, as larger coupling strengths facilitate synchronization and therefore reduce the critical pinning strength. In pinning control of network synchronization, another important concern is the dependence of the network controllability on the fraction of pinning nodes. Typically, 

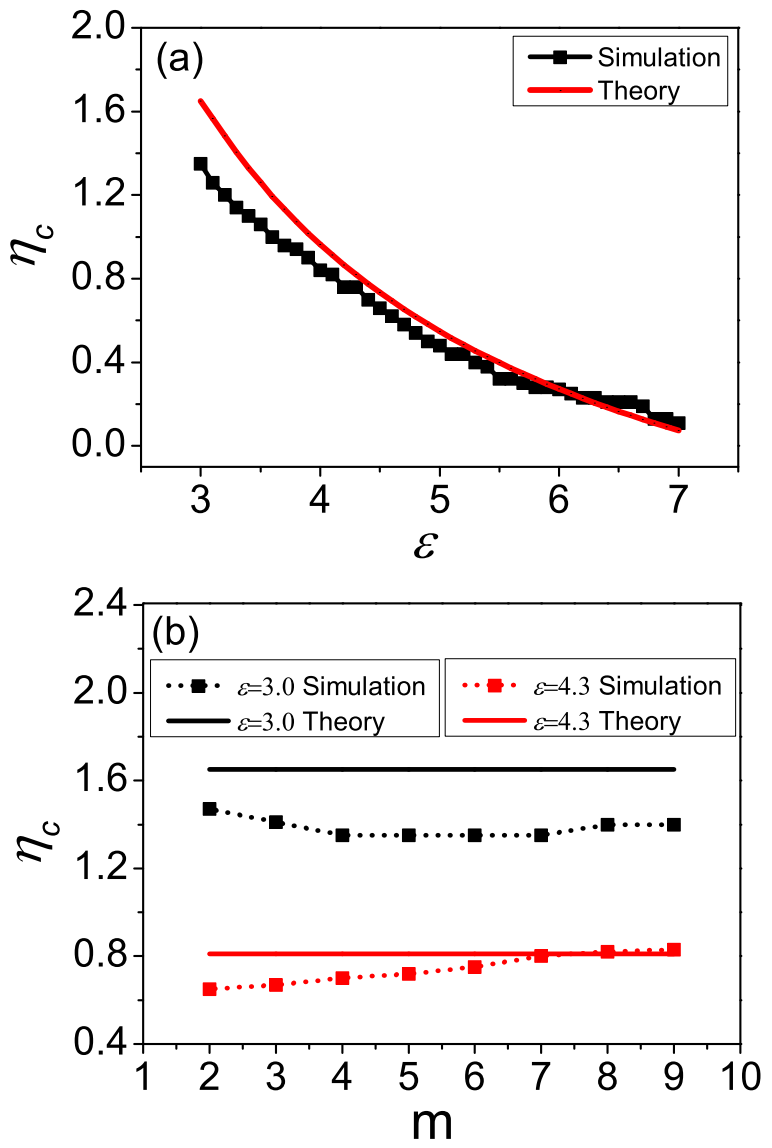

FIG. 2. For the globally connected network, the variation of $\eta_{c}$ with respect to $\varepsilon$ and $m$. (a) For $m=5, \eta_{c}$ vs $\varepsilon$. (b) For $\varepsilon=3.0$ and $4.3, \eta_{c}$ vs $m$. Squares are the results obtained from numerical simulations and solid lines are the results from theoretical predications.

with the increase of the number of pinning nodes, the critical pinning strength is gradually decreased [35-38]. Fixing $\varepsilon=$ 3.0, we plot in Fig. 2(b) the variation of $\eta_{c}$ with respect to $m$. We see that the value of $\eta_{c}$ is almost kept unchanged by varying $m$. The same phenomenon is also observed for other coupling strengths [e.g., the results for $\varepsilon=4.3$ shown in Fig. 2(b)].

\section{B. Pinning CS in ring-structure network}

We next investigate the control of CS in a ring-structure network, in which each oscillator is coupled to its nearest neighbors. Still, we set the network size as $N^{\prime}=10$ and adopt the chaotic Lorenz oscillator as the nodal dynamics and use the coupling function $\mathbf{H}([\mathbf{x}, \mathbf{y}, \mathbf{z}])=[0, x, 0]^{T}$. Different from globally connected network, in ring-structure network the system dynamics is dependent on the pinning locations. For instance, when $m=2$ nodes are pinned, we have nine different pinning realizations in total. According to the network symmetries, these realizations can be divided into two groups. In group one, the network satisfies the reflection symmetry $S_{1}$ and nodes in the original network are partitioned into five symmetric pairs. An example of this kind of network is shown in Fig. 3(a1), where the paired nodes are $(1,10),(2,9)$,
$(3,8),(4,7)$, and $(5,6)$. In group two, the network satisfies the reflection symmetry $S_{2}$, and nodes in the original network are partitioned into four symmetric pairs and two isolated nodes. An example of this kind of network is shown in Fig. 3(b1), where the paired nodes are $(2,10),(3,9),(4,8)$, and $(5,7)$. Since the network dynamics is dependent on the pinning locations, the performance of pinning control therefore should be evaluated case by case.

For the ring-structure network, the critical coupling strength for global synchronization is $\varepsilon_{c} \approx 43.4$, which can be obtained from the standard MSF method. In numerical simulations, we set $\varepsilon=20$, so as to make the network stay in the desynchronization state without pinnings. Pinning nodes 3 and 8, we plot in Fig. 3(a2) the synchronization error between the paired nodes, $\delta x_{i j}=\left|x_{i}-x_{j}\right|$, with respect to the pinning strength $\eta$. We see that with the increase of $\eta$, the synchronization errors between the paired nodes are gradually decreased and reach 0 all together at $\eta_{c} \approx 0.87$. To have more details about the system dynamics, we also plot in Fig. 3(a2) the variations of the global synchronization error, $\langle\delta x\rangle_{\text {net }}=$ $\sum_{i=1}^{N^{\prime}}\left|x_{i}-\hat{x}\right| / N^{\prime}$ (with $\hat{x}=\sum_{i=1}^{N^{\prime}} x_{i} / N^{\prime}$ the averaged state of all nodes in the original network), and the synchronization error between the averaged state of the pinning nodes and the controller, $\delta x_{T}=\left|x_{T}-\bar{x}\right|$. We see that both $\delta x_{\text {net }}$ and $\delta x_{T}$ remain at large values at $\eta_{c}$. As synchronization is achieved between only the paired nodes, the network thus is controlled to the CS state consisting of five synchronous clusters (each cluster contains two nodes). The dependence of $\eta_{c}$ on $\varepsilon$ is plotted in Fig. 3(a3). We see that similar to the results of globally connected network [Fig. 2(a)], $\eta_{c}$ is decreased monotonically as $\varepsilon$ increases.

Fixing $\varepsilon=25$, we plot in Fig. 3(b) the results for pinning nodes 3 and 9. Figure 3(b2) shows that the paired nodes are synchronized for $\eta>\eta_{c} \approx 0.62$. Again, synchronization is restricted to the paired nodes only, and the pinning nodes are not synchronized with the controller. The network therefore is controlled to a CS state consisting of four synchronous clusters, while each cluster contains two nodes. The dependence of $\eta_{c}$ on $\varepsilon$ is plotted in Fig. 3(b3). We see that $\eta_{c}$ is decreased monotonically with $\varepsilon$. Figure 3 suggests that different from globally connected network, in ring-structure network both the CS state and the critical pinning strength are affected by the pinning locations.

To further exemplify the control of CS in the ring-structure network, we add pinnings on $m=5$ nodes equally spaced on the ring, as depicted in Fig. 4(a). The network structure now satisfies the discrete rotational symmetry $S_{3}$ and the network nodes are divided into two symmetric groups: $V_{1}=$ $\{1,3,5,7,9\}$ and $V_{2}=\{2,4,6,8,10\}$. The variation of the synchronization error of the pinning nodes, $\langle\delta x\rangle_{1}$, with respect to the pinning strength $\eta$ is plotted in Fig. 4(b). We see that $\langle\delta x\rangle_{1}$ reaches 0 at $\eta_{c} \approx 0.35$. In Fig. 4(b), we also present the variations of the synchronization error of the unpinning nodes, $\langle\delta x\rangle_{2}$, with respect to $\eta$. We see that $\langle\delta x\rangle_{2}$ also reaches 0 at $\eta_{c}$. Meanwhile, the results of the global synchronization error, $\langle\delta x\rangle_{\text {net }}$, and the synchronization error between the pinning nodes and the controller, $\delta x_{T}$, show that they keep at large values at $\eta_{c}$, but are decreased to 0 at $\eta_{g} \approx 0.85$. Therefore, the network is controlled to a CS state consisting of two 

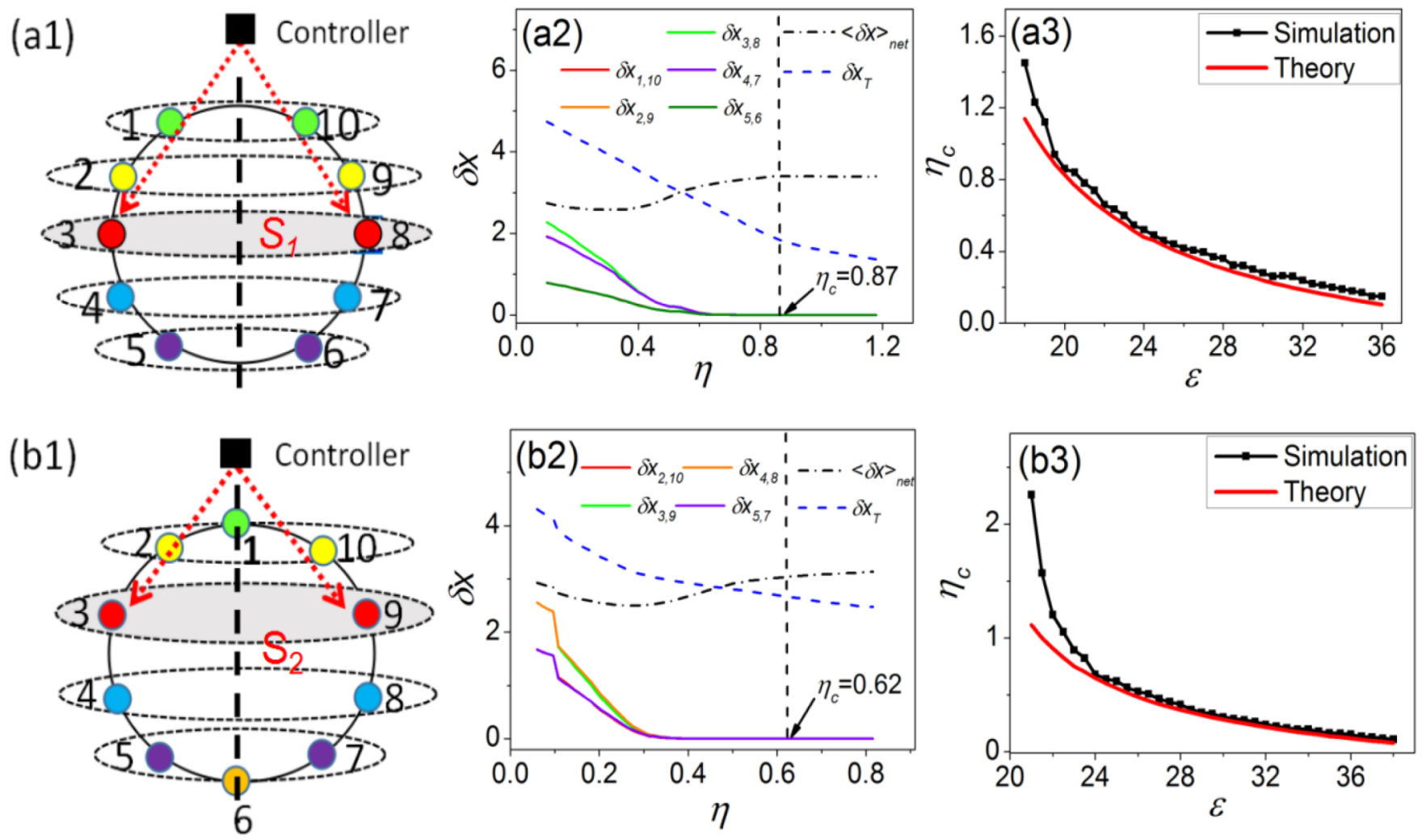

FIG. 3. Pinning control of CS in ring-structure network. The network size is $N^{\prime}=10$ and pinnings are added on $m=2$ nodes. (a1) Pinning nodes 3 and 8 , the network structure satisfies the reflection symmetry $S_{1}$. The network contains five symmetric node pairs: $(1,10),(2,9),(3,8)$, $(4,7)$, and $(5,6)$. (a2) For $\varepsilon=20$, the variation of the synchronization error between the paired nodes, $\delta x_{i j}$, with respect to the pinning strength $\eta$. All the paired nodes are synchronized at the same critical pinning strength, $\eta_{c} \approx 0.87$. $\langle\delta x\rangle_{\text {net }}$ represents the global synchronization error. $\delta x_{T}$ is the synchronization error between the averaged state of the pinning nodes and the controller. Note that $\delta x_{2,9} \approx \delta x_{4,7}$ and $\delta x_{1,10} \approx \delta x_{5,6}$ for $\eta<\eta_{c}$. (a3) The dependence of $\eta_{c}$ on $\varepsilon$. (b1) Pinning nodes 3 and 9, the network structure satisfies the reflection symmetry $S_{2}$. The network contains four symmetric node pairs: $(2,10),(3,9),(4,8)$, and (5,7). (b2) For $\varepsilon=25$, the variations of $\delta x_{i j}$, $\left\langle\delta x_{\text {net }}\right\rangle$ and $\delta x_{T}$ with respect to $\eta$. All the paired nodes are synchronized at $\eta_{c} \approx 0.62$. Note that $\delta x_{3,9} \approx \delta x_{4,8}$ and $\delta x_{2,10} \approx \delta x_{5,7}$ for $\eta<\eta_{c}$. (b3) $\eta_{c}$ vs $\varepsilon$. In (a3) and (b3), the solid lines are the results from theoretical predications.

synchronous clusters in the region of $\eta \in\left(\eta_{c}, \eta_{g}\right)$ : one is formed by the pinning nodes and the other is formed by the unpinning nodes. Global synchronization is achieved at $\eta_{g}$, where the two clusters are synchronized and are also synchronized with the controller. This phenomenon is different from that of globally connected network where the unpinning nodes are always desynchronized (see Fig. 1), and is also different from the case of $m=2$ pinning nodes in ring-structure network where the network is not globally synchronized even under very large pinning strengths (see Fig. 3).

Figures 3 and 4 show the flexibility of pinning control in generating CS in ring-structure network. More specifically, by varying the number of pinning nodes or changing the pinning locations, we see that the same network can be controlled to different CS states. Furthermore, different from the results of globally connected network (see Figs. 1 and 2 ), we see that in ring-structure network the critical pinning strength is dependent on the specific CS state to be controlled to [see Figs. 3(a2) and 3(b2)] and, interestingly, the group of unpinning nodes also can be synchronized (see Fig. 4). These numerical observations arouse our interest about the dynamical mechanism generating CS, which is explored in the following section.

\section{THEORETICAL ANALYSIS}

\section{A. Symmetry-based stability analysis}

The stabilities of the CS states under pinnings can be analyzed by the method of symmetry-based stability analysis $[19,21,23,25,28,31]$, with the details as follows. Assume that the enlarged network (combining the original network and the controller) satisfies symmetry $S$, we are then able to divide the network nodes into $M$ clusters. The set of nodes in cluster $l$ is denoted as $V_{l}$, with $l=1, \ldots, M$ the cluster index, and the size of the $l$ th cluster is denoted as $n_{l}$. Following Refs. [23,25], here symmetric cluster is defined as the set of nodes whose permutations do not change the network structure. If the permutation of the nodes within one cluster is dependent on the permutation of nodes within another cluster, we say that the two clusters are intertwined with each other. (Please note that due to the feature of pinning coupling, the controller is not symmetric with any other node in the network, i.e., it belongs to an isolated node.) Each partition of the symmetric clusters supports a unique CS state. That is, if we artificially set the initial conditions of the nodes within each cluster as identical, then, without noise perturbations, the network will always stay on this artificially prepared CS state. Denoting 


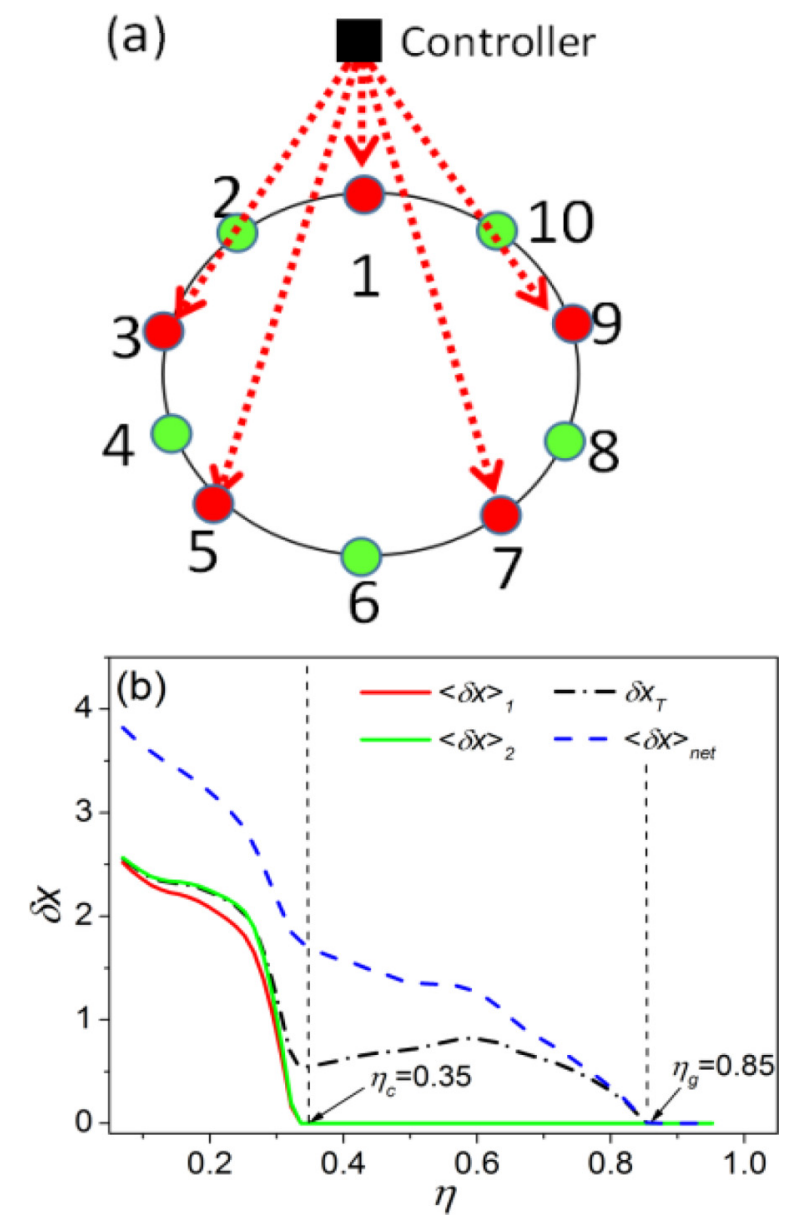

FIG. 4. Pinning $m=5$ nodes in a ring-structure network of $N^{\prime}=$ 10 nodes. (a) The network structure and the pinning configuration. The network nodes are partitioned into two symmetric groups, $V_{1}=\{1,3,5,7,9\}$ (the pinning nodes) and $V_{2}=\{2,4,6,8,10\}$ (the unpinning nodes), and an isolated node (the controller). (b) The variations of the synchronization errors of the pinning nodes $\left(\langle\delta x\rangle_{1}\right)$, the unpinning nodes $\left(\langle\delta x\rangle_{2}\right)$, all nodes in the original network $\left(\langle\delta x\rangle_{\text {net }}\right)$, and between the pinning nodes and the controller $\left(\delta x_{T}\right)$ with respect to the pinning strength $\eta .\langle\delta x\rangle_{1}$ and $\langle\delta x\rangle_{2}$ reach 0 at $\eta_{c} \approx 0.35 .\langle\delta x\rangle_{\text {net }}$ and $\delta x_{T}$ reach 0 at $\eta_{g} \approx 0.85$.

$\mathscr{X}_{l}$ as the common manifold (synchronization manifold) of all nodes in the $l$ th cluster (i.e., $\mathbf{x}_{i}=\mathscr{X}_{l}$ for $i \in V_{l}$ ), the evolution of $\mathscr{X}_{l}$ is governed by equation

$$
\dot{\mathscr{X}}_{l}=\mathbf{F}\left(\mathscr{X}_{l}\right)+\varepsilon \sum_{l^{\prime}=1}^{M} w_{l l^{\prime}} \mathbf{H}\left(\mathscr{X}_{l^{\prime}}\right),
$$

with $l, l^{\prime}=1, \ldots, M$ the cluster indices, and $w_{l l^{\prime}}=$ $\sum_{j \in V_{l^{\prime}}} c_{i j} / n_{l}$ the integrated coupling strength that node $i$ in cluster $l$ is received from all nodes in cluster $l^{\prime}$. The diagonal elements are set as $w_{l l}=-\sum_{l^{\prime}} w_{l l^{\prime}}$. The coupling matrix $\mathbf{W}_{M \times M}$ defines a weighted network containing only $M$ nodes, i.e., the quotient network $[11,25]$, which governs the dynamics of the CS state in the absence of noise perturbations, i.e., the dynamics of the CS manifold.

We proceed to analyze the stability of the CS state described by Eq. (3) under noise perturbations. Let $\delta \mathbf{x}_{i}=\mathbf{x}_{i}-\mathscr{X}_{l}$ be infinitesimal perturbations added on oscillator $i$; then, whether the CS state is stable is determined by the following set of variational equations [obtained by linearizing Eq. (2) around $\mathscr{X}_{i}$ :

$$
\delta \dot{\mathbf{x}}_{i}=D \mathbf{F}\left(\mathscr{X}_{l}\right) \delta \mathbf{x}_{i}+\varepsilon \sum_{l^{\prime}=1}^{M} \sum_{j \in V_{l^{\prime}}} c_{i j} D \mathbf{H}\left(\mathscr{X}_{l^{\prime}}\right) \delta \mathbf{x}_{j},
$$

with $D \mathbf{F}$ and $D \mathbf{H}$ the Jacobin matrices. On the right-hand-side of Eq. (4), the second term refers to the couplings that node $i$ receives from all other nodes in the network, and the coupling signals are grouped according to the cluster partitions. To make the CS stable, the necessary condition is that $\delta \mathbf{x}$ damps to 0 in all directions transverse to the synchronization manifolds defined by Eq. (3).

Denoting $\mathbf{R}_{S}$ as the permutation matrix associated with the network symmetry $S$, we can analyze the time evolution of the perturbations [as described by Eq. (4)] in the mode space spanned by the eigenvectors of $\mathbf{R}_{S}$. The permutation matrix $\mathbf{R}_{S}$ is constructed according to the cluster partitions, as follows. If nodes $i$ and $j$ are symmetric in the enlarged network, we set $r_{i j}=r_{j i}=1$, otherwise $r_{i j}=r_{j i}=0$; if node $i$ does not belong to any cluster, i.e., it is an isolated node, we set $r_{i i}=1$, while setting $r_{i j}=0$ for $i \neq j$. Denoting $\mathbf{T}_{S}$ as the transformation matrix constructed by the eigenvectors of $\mathbf{R}_{S}$, we apply it to the coupling matrix $\mathbf{C}$ and can obtain the blocked matrix

$$
\mathbf{C}^{\prime}=\mathbf{T}_{S}^{-1} \mathbf{C} \mathbf{T}_{S}=\left(\begin{array}{cc}
\mathbf{D} & 0 \\
0 & \mathbf{B}
\end{array}\right)
$$

The submatrix $\mathbf{D}=\left\{d_{l l^{\prime}}\right\}$ has the dimensions $M \times M$, which characterizes the space of the synchronization manifolds [i.e., the dynamics described by Eq. (3)]. The submatrix $\mathbf{B}=\left\{b_{i j}\right\}$ has the dimensions $(N-M) \times(N-M)$ and characterizes the space transverse to the synchronization manifolds. (Besides dimensions, the submatrix $\mathbf{D}$ is also distinguished from B by owing the null eigenvalue $[19,25]$.)

We would like to note that the role of the network symmetry, as shown in Eq. (5), is to separate the transverse space from the synchronous space, so that the stabilities of the clusters can be analyzed separately. Because $\mathbf{C}$ and $\mathbf{C}^{\prime}$ are similar matrices, they share the same set of eigenvalues. Therefore, by the symmetry-based transformation, the eigenvalues are actually divided into two groups: one is associated with the transverse space and the other one is associated with the synchronous space. This treatment is different from the method of irreducible representations (IRRs) proposed in Ref. [23] in that (1) the transformation matrix $\mathbf{T}_{S}$ is constructed from the eigenvectors of the permutation matrix $\mathbf{R}_{S}$ and (2) the submatrix $\mathbf{B}$ is not diagonalized.

Let $\quad \Delta \mathbf{Y}=\mathbf{T}_{S}^{-1} \Delta \mathbf{X}=\left(\delta \mathbf{y}_{1}^{s}, \ldots, \delta \mathbf{y}_{M}^{s}, \delta \mathbf{y}_{1}^{t}, \ldots, \delta \mathbf{y}_{N-M}^{t}\right)^{T}$ be the perturbations in the mode space, with $\delta \mathbf{y}_{l}^{s}$ the mode parallel to $\mathscr{X}_{l}$ (i.e., the synchronous mode of cluster $l$ ) and $\delta \mathbf{y}_{i}^{t}$ the $i$ th mode in the $(N-M)$-dimensional transverse space. Then, the dynamics of the synchronous modes are governed by equations

$$
\delta \dot{\mathbf{y}}_{l}^{s}=D \mathbf{F}\left(\mathscr{X}_{l}\right) \delta \mathbf{y}_{l}^{s}+\varepsilon \sum_{l^{\prime}=1}^{M} d_{l l^{\prime}} D \mathbf{H}\left(\mathscr{X}_{l^{\prime}}\right) \delta \mathbf{y}_{l^{\prime}}^{s},
$$


with $l, l^{\prime}=1, \ldots, M$ the cluster indices, and the dynamics of the transverse modes are governed by equations

$$
\delta \dot{\mathbf{y}}_{i}^{t}=D \mathbf{F}\left(\mathscr{X}_{l}\right) \delta \mathbf{y}_{i}^{t}+\varepsilon \sum_{j=1}^{N-M} b_{i j} D \mathbf{H}\left(\mathscr{X}_{l^{\prime}}\right) \delta \mathbf{y}_{j}^{t},
$$

with $\mathscr{X}_{l}$ and $\mathscr{X}_{l^{\prime}}$ the synchronization manifolds of the clusters containing nodes $i$ and $j$, respectively. To make the CS state stable, now the necessary conditions become that $\delta \mathbf{y}_{i}^{t}$ damps to 0 with time for all $N-M$ transverse modes, i.e., all the conditional Lyapunov exponents in the transverse space should be negative. Therefore, to evaluate whether a CS state is stable, in principle we need to solve numerically Eqs. (3) and (7) together.

The above analysis can be simplified if the clusters are not intertwined. According to the partition of the node clusters, we can divide the transverse space into $M$ transverse subspaces, where each subspace stands for a specific cluster. For the general networks, these subspaces are intertwined (coupled) with each other. However, for some special networks (e.g., the case of globally connected networks), the transverse subspaces can be decoupled from each other. In such a case, the transverse matrix can be expressed as a direct sum of $M$ submatrices $\mathbf{B}=\sum_{l=1}^{M} \oplus \mathbf{B}^{l}$, with $\mathbf{B}^{l}=\left\{b_{i j}^{l}\right\}$ the $\left(n_{l}-1\right)$ dimensional submatrix associated with cluster $l$. Denoting $\delta \mathbf{y}_{i}^{l}$ as the $i$ th transverse modes of $\mathbf{B}^{l}$, its evolution is governed by the equation

$$
\delta \dot{\mathbf{y}}_{i}^{l}=D \mathbf{F}\left(\mathscr{X}_{l}\right) \delta \mathbf{y}_{i}^{l}+\varepsilon D \mathbf{H}\left(\mathscr{X}_{l}\right) \sum_{j=1}^{n_{l}-1} b_{i j}^{l} \delta \mathbf{y}_{j}^{l} .
$$

Transforming Eq. (8) into the mode space spanned by the eigenvectors of $\mathbf{B}^{l}$, we have the set of variational equations,

$$
\delta \dot{\mathbf{z}}_{i}^{l}=\left[D \mathbf{F}\left(\mathscr{X}_{l}\right)+\varepsilon \lambda_{i}^{l} D \mathbf{H}\left(\mathscr{X}_{l}\right)\right] \delta \mathbf{z}_{i}^{l},
$$

with $0>\lambda_{1}^{l} \geqslant \lambda_{2}^{l} \geqslant \cdots \geqslant \lambda_{n_{l}-1}^{l}$ the eigenvalues of $\mathbf{B}^{l}$. Equation (9) serves as the master equation of the transverse modes for cluster $l$, which is similar to the one used in MSF except that here the synchronization manifold is defined on the cluster. Therefore, in evaluating the stability of the CS state in decoupled clusters, we need to solve only Eqs. (3) and (9), which is simplified as compared to solving Eqs. (3) and (7) for the case of intertwined clusters.

The analysis could be simplified further if the synchronization manifolds of the clusters fall onto attractors similar to that of an isolated oscillator [41]. Specifically, if the statistical distributions of $D \mathbf{F}\left(\mathscr{X}_{l}\right)$ and $D \mathbf{H}\left(\mathscr{X}_{l}\right)$ are similar to that of the isolated oscillator, then Eq. (9) can be treated as the unified master equation for all clusters. (This happens normally when the coupling strength is very weak, or the coupling strength is very strong such that the whole system is at the boundary of global synchronization. Even for moderate coupling strengths, this could also be a good approximation if the dynamics of the oscillators are robust to couplings, e.g., the chaotic Lorenz oscillators adopted in the present work [31].) In such a case, we can replace $\mathscr{X}_{l}$ with $\mathscr{X}_{T}$ (the manifold of the controller), and the stability of the CS state is solely determined by the eigenvalues of $\mathbf{B}$. Moreover, if the clusters are decoupled from each other, the stabilities of the synchronization clusters can be analyzed separately by the same master equation.

Given that the statistical properties of the cluster synchronization manifolds can be approximated by the manifold of the isolated oscillator, we have the following criteria on the stability of the CS states. If the clusters are intertwined with each other, the necessary condition for the CS state to be stable is that the largest Lyapunov exponent of Eq. (7), $\Lambda$, is negative for all $N-M$ transverse modes. Defining $\sigma \equiv-\varepsilon \lambda$ as the generic coupling strength, we can obtain numerically the variation of $\Lambda$ with respect to $\sigma$. For the chaotic Lorenz oscillators and coupling function adopted in the present work, previous studies show that $\Lambda$ is negative when $\sigma$ exceeds the threshold $\sigma_{c}$ [14-16], with $\sigma_{c}$ a value to be determined by numerical simulations. To make the CS state stable, now the condition becomes that $\sigma_{i}^{B}=-\varepsilon \lambda_{i}^{B}$ falls in the stable region of $\Lambda<0$ for all the transverse modes. Here, $0>\lambda_{1}^{B} \geqslant \lambda_{2}^{B} \geqslant$ $\cdots \geqslant \lambda_{N-M}^{B}$ are eigenvalue of $\mathbf{B}$. The criteria for generating $\mathrm{CS}$ in intertwined clusters therefore is

$$
-\varepsilon \lambda_{1}^{B}>\sigma_{c}
$$

If the clusters can be decoupled from each other, the stabilities of the clusters can be evaluated individually. The condition for the $l$ th cluster to be stable is

$$
-\varepsilon \lambda_{1}^{l}>\sigma_{c}
$$

We note that due to the pinning couplings, both $\lambda_{1}^{B}$ and $\lambda_{1}^{l}$ could be dependent on $\eta$.

After reaching CS, the further increase of $\eta$ may result in global synchronization. The critical pinning strength for global synchronization can be analyzed by Eq. (6). Specifically, let $0=\lambda_{1}^{D}>\lambda_{2}^{D} \geqslant \cdots \geqslant \lambda_{M}^{D}$ be the eigenvalues of $\mathbf{D}$; then, the condition for global network synchronization is

$$
-\varepsilon \lambda_{2}^{D}>\sigma_{c}
$$

If $\lambda_{2}^{D}>\lambda_{1}^{B}$, this condition will be identical to the condition of global synchronization as obtained by the standard MSF method. In such a case, CS could be generated. However, if $\lambda_{2}^{D}<\lambda_{1}^{B}$, the condition for global synchronization will be determined by $\lambda_{1}^{B}$. In such a case, no CS state will be generated and the network will reach global synchronization directly by increasing the pinning strength. Equation (12) also suggests that if $\lambda_{2}^{D}$ is independent of $\eta$, the network can never be globally synchronized by increasing $\eta$.

\section{B. The theoretical results for globally connected network}

By the above method of symmetry-based stability analysis, we now are able to analyze the stabilities of the CS states observed in numerical simulations. We start by analyzing the control of CS in a globally connected network (the numerical results are shown in Figs. 1 and 2). For $N^{\prime}=10$ and $m=5$, 
the coupling matrix of the enlarged network reads

$$
\mathbf{C}=\left(\begin{array}{ccccccccccc}
-1-\eta & \beta & \beta & \beta & \beta & \beta & \beta & \beta & \beta & \beta & \eta \\
\beta & -1-\eta & \beta & \beta & \beta & \beta & \beta & \beta & \beta & \beta & \eta \\
\beta & \beta & -1-\eta & \beta & \beta & \beta & \beta & \beta & \beta & \beta & \eta \\
\beta & \beta & \beta & -1-\eta & \beta & \beta & \beta & \beta & \beta & \beta & \eta \\
\beta & \beta & \beta & \beta & -1-\eta & \beta & \beta & \beta & \beta & \beta & \eta \\
\beta & \beta & \beta & \beta & \beta & -1 & \beta & \beta & \beta & \beta & 0 \\
\beta & \beta & \beta & \beta & \beta & \beta & -1 & \beta & \beta & \beta & 0 \\
\beta & \beta & \beta & \beta & \beta & \beta & \beta & -1 & \beta & \beta & 0 \\
\beta & \beta & \beta & \beta & \beta & \beta & \beta & \beta & -1 & \beta & 0 \\
\beta & \beta & \beta & \beta & \beta & \beta & \beta & \beta & \beta & -1 & 0 \\
0 & 0 & 0 & 0 & 0 & 0 & 0 & 0 & 0 & 0 & 0
\end{array}\right),
$$

with $\beta=1 /\left(N^{\prime}-1\right)=1 / 9$. For convenience, here we have reordered the nodes such that the pinning nodes are of index from 1 to $m$, and the controller is of index $N$. Without pinnings, all nodes belong to the same cluster, as the mutation of any two nodes does not affect the network dynamics. However, when pinnings are introduced, the network symmetry will be degenerated and the nodes are divided into two symmetric clusters, $V_{1}=\{1,2,3,4,5\}$ (pinning nodes), $V_{2}=\{6,7,8,9,10\}$ (unpinning nodes), and an isolated node (the controller). According to the partition of the symmetric clusters, we can construct the following permutation matrix:

$$
\mathbf{R}_{S}=\left(\begin{array}{lllllllllll}
0 & 1 & 1 & 1 & 1 & 0 & 0 & 0 & 0 & 0 & 0 \\
1 & 0 & 1 & 1 & 1 & 0 & 0 & 0 & 0 & 0 & 0 \\
1 & 1 & 0 & 1 & 1 & 0 & 0 & 0 & 0 & 0 & 0 \\
1 & 1 & 1 & 0 & 1 & 0 & 0 & 0 & 0 & 0 & 0 \\
1 & 1 & 1 & 1 & 0 & 0 & 0 & 0 & 0 & 0 & 0 \\
0 & 0 & 0 & 0 & 0 & 0 & 1 & 1 & 1 & 1 & 0 \\
0 & 0 & 0 & 0 & 0 & 1 & 0 & 1 & 1 & 1 & 0 \\
0 & 0 & 0 & 0 & 0 & 1 & 1 & 0 & 1 & 1 & 0 \\
0 & 0 & 0 & 0 & 0 & 1 & 1 & 1 & 0 & 1 & 0 \\
0 & 0 & 0 & 0 & 0 & 1 & 1 & 1 & 1 & 0 & 0 \\
0 & 0 & 0 & 0 & 0 & 0 & 0 & 0 & 0 & 0 & 1
\end{array}\right),
$$

and, by the eigenvectors of $\mathbf{R}_{S}$, obtain the transformation matrix

$$
\mathbf{T}_{S}=\left(\begin{array}{ccccccccccc}
-0.47 & 0.09 & 0.71 & -0.27 & 0 & 0 & 0 & 0 & 0 & -0.45 & 0 \\
-0.47 & 0.09 & -0.71 & -0.27 & 0 & 0 & 0 & 0 & 0 & -0.45 & 0 \\
0.39 & 0.75 & 0 & 0.3 & 0 & 0 & 0 & 0 & 0 & -0.45 & 0 \\
0.63 & -0.41 & 0 & -0.48 & 0 & 0 & 0 & 0 & 0 & -0.45 & 0 \\
-0.09 & -0.51 & 0 & 0.73 & 0 & 0 & 0 & 0 & 0 & -0.45 & 0 \\
0 & 0 & 0 & 0 & 0.71 & -0.27 & -0.47 & 0.09 & 0 & 0 & -0.45 \\
0 & 0 & 0 & 0 & -0.71 & -0.27 & -0.47 & 0.09 & 0 & 0 & -0.45 \\
0 & 0 & 0 & 0 & 0 & 0.3 & 0.4 & 0.75 & 0 & 0 & -0.45 \\
0 & 0 & 0 & 0 & 0 & -0.48 & 0.63 & -0.41 & 0 & 0 & -0.45 \\
0 & 0 & 0 & 0 & 0 & 0.73 & -0.09 & -0.51 & 0 & 0 & -0.45 \\
0 & 0 & 0 & 0 & 0 & 0 & 0 & 0 & 1 & 0 & 0
\end{array}\right) .
$$

By the operation $\mathbf{C}^{\prime}=\mathbf{T}_{S}^{-1} \mathbf{C} \mathbf{T}_{S}$, the coupling matrix $\mathbf{C}$ can be transformed into the blocked form as shown in Eq. (5), where the two submatrices are

$$
\mathbf{D}=\left(\begin{array}{ccc}
0 & 0 & 0 \\
-\sqrt{5} \eta & -\frac{5}{9}-\eta & \frac{5}{9} \\
0 & \frac{5}{9} & -\frac{5}{9}
\end{array}\right)
$$

and

$$
\mathbf{B}=\left(\begin{array}{cc}
\mathbf{B}^{1} & 0 \\
0 & \mathbf{B}^{2}
\end{array}\right)=\left(\begin{array}{cccccccc}
-\gamma-\eta & 0 & 0 & 0 & 0 & 0 & 0 & 0 \\
0 & -\gamma-\eta & 0 & 0 & 0 & 0 & 0 & 0 \\
0 & 0 & -\gamma-\eta & 0 & 0 & 0 & 0 & 0 \\
0 & 0 & 0 & -\gamma-\eta & 0 & 0 & 0 & 0 \\
0 & 0 & 0 & 0 & -\gamma & 0 & 0 & 0 \\
0 & 0 & 0 & 0 & 0 & -\gamma & 0 & 0 \\
0 & 0 & 0 & 0 & 0 & 0 & -\gamma & 0 \\
0 & 0 & 0 & 0 & 0 & 0 & 0 & -\gamma
\end{array}\right),
$$


with $\gamma=N^{\prime} /\left(N^{\prime}-1\right)=10 / 9$. Since the null eigenvalue is contained in $\mathbf{D}$, the synchronous and transverse subspaces thus are spanned by the eigenvectors of $\mathbf{D}$ and $\mathbf{B}$, respectively. The submatrix $\mathbf{B}^{1}$ is of dimensions $4 \times 4$, which characterizes the transverse space of cluster 1 . The submatrix $\mathbf{B}^{2}$ is also of dimensions $4 \times 4$, which characterizes the transverse space of cluster 2. We see from Eq. (17) that the two submatrices are decoupled from each other, indicating that their stabilities can be analyzed separately. As such, the stabilities of the two clusters can be analyzed by Eq. (9). Furthermore, Eq. (17) shows that the variation of $\eta$ affects only $\mathbf{B}^{1}$, while $\mathbf{B}^{2}$ remains unchanged.

Replacing $\mathscr{X}_{l}$ with $\mathscr{X}_{T}$ in Eq. (9), we have the master equation

$$
\delta \dot{\mathbf{z}}=\left[D \mathbf{F}\left(\mathscr{X}_{T}\right)+\sigma D \mathbf{H}\left(\mathscr{X}_{T}\right)\right] \delta \mathbf{z},
$$

with $\sigma=-\varepsilon \lambda_{i}^{l}$ and $\lambda_{i}^{l}$ the eigenvalues of $\mathbf{B}^{1}$ or $\mathbf{B}^{2}$. Numerical results show that for the Lorenz oscillator and coupling function used here, the largest Lyapunov exponent of Eq. (18) is negative for $\sigma>\sigma_{c} \approx 8.3$. As all eigenvalues of $\mathbf{B}^{1}$ equal $-(\eta+\gamma)$, we therefore have from Eq. (11) the critical pinning strength,

$$
\eta_{c}=\sigma_{c} / \varepsilon-\gamma .
$$

For $\varepsilon=5.0$, Eq. (19) gives $\eta_{c} \approx 0.55$, which agrees well with the numerical result shown in Fig. 1. According to Eq. (19), we again plot in Fig. 2(a) the variation of $\eta_{c}$ with respect to $\varepsilon$. We see that the theoretical predictions agree with the numerical results over the scanned region. Equation (19) also suggests that the critical pinning strength $\eta_{c}$ is independent of the number of pinning nodes $m$. This explains the numerical results plotted in Fig. 2(b).

As $\mathbf{B}^{2}$ is independent of $\eta$, the value of $\lambda_{1}^{2}$ is not affected by $\eta$. As a result of this, the second cluster (the group of unpinning nodes) cannot be synchronized by increasing $\eta$, and therefore the whole network always remains in the desynchronization state, as shown in Fig. 1.

It is straightforward to generalize the above results to a globally connected network of any size. For a network of size $N^{\prime}$, the eigenvalues of $\mathbf{B}^{1}$ will be $-\left[\eta+N^{\prime} /\left(N^{\prime}-1\right)\right]$, which, according to Eq. (11), give the critical pinning strength $\eta_{c}=$ $\left[\sigma_{c} / \varepsilon-N^{\prime} /\left(N^{\prime}-1\right)\right]$ for synchronizing the pinning nodes. We see that despite the variation of the network size, the critical pinning strength is always independent of the number of pinning nodes.

\section{The theoretical results for ring-structure network}

We proceed to analyze the stabilities of the CS states controlled in the ring-structure network. For the pinning configuration shown in Fig. 3(a1) (i.e., pining nodes 3 and 8 ), the enlarged network satisfies the reflection symmetry $S_{1}$ and nodes in the original network (without the controller) are divided into five symmetric pairs: $(1,10),(2,9),(3,8)$, $(4,7)$, and $(5,6)$. Regarding each symmetric pair as a cluster, the enlarged network thus consists of $M=5$ clusters and an isolated node (the controller). Different from the situation of globally connected network, here the clusters are intertwined with each other. More specifically, to keep the network dynamics unchanged, the paired nodes should be permuted all together. As discussed in Sec. IV A, for intertwined clusters, the stabilities of the CS state should be evaluated according to Eq. (7).

From the network symmetry $S_{1}$, we can construct the permutation matrix

$$
\mathbf{R}_{S 1}=\left(\begin{array}{ccccccccccc}
0 & 0 & 0 & 0 & 0 & 0 & 0 & 0 & 0 & 1 & 0 \\
0 & 0 & 0 & 0 & 0 & 0 & 0 & 0 & 1 & 0 & 0 \\
0 & 0 & 0 & 0 & 0 & 0 & 0 & 1 & 0 & 0 & 0 \\
0 & 0 & 0 & 0 & 0 & 0 & 1 & 0 & 0 & 0 & 0 \\
0 & 0 & 0 & 0 & 0 & 1 & 0 & 0 & 0 & 0 & 0 \\
0 & 0 & 0 & 0 & 1 & 0 & 0 & 0 & 0 & 0 & 0 \\
0 & 0 & 0 & 1 & 0 & 0 & 0 & 0 & 0 & 0 & 0 \\
0 & 0 & 1 & 0 & 0 & 0 & 0 & 0 & 0 & 0 & 0 \\
0 & 1 & 0 & 0 & 0 & 0 & 0 & 0 & 0 & 0 & 0 \\
1 & 0 & 0 & 0 & 0 & 0 & 0 & 0 & 0 & 0 & 0 \\
0 & 0 & 0 & 0 & 0 & 0 & 0 & 0 & 0 & 0 & 1
\end{array}\right),
$$

and, by the operation $\mathbf{C}^{\prime}=\mathbf{T}_{S 1}^{-1} \mathbf{C} \mathbf{T}_{S 1}$ (with $\mathbf{T}_{S 1}$ the transformation matrix constructed from the eigenvectors of $\mathbf{R}_{S 1}$ ), transform the coupling matrix into the blocked form shown in Eq. (5). The submatrix governing the dynamics of the synchronization manifolds is

$$
\mathbf{D}=\left(\begin{array}{cccccc}
-1 & \frac{1}{2} & 0 & 0 & \frac{1}{2} & 0 \\
\frac{1}{2} & -\eta-1 & \frac{1}{2} & 0 & 0 & \sqrt{2} \eta \\
0 & \frac{1}{2} & -1 & \frac{1}{2} & 0 & 0 \\
0 & 0 & \frac{1}{2} & -\frac{1}{2} & 0 & 0 \\
\frac{1}{2} & 0 & 0 & 0 & -\frac{1}{2} & 0 \\
0 & 0 & 0 & 0 & 0 & 0
\end{array}\right),
$$

and the submatrix governing the dynamics of the transverse modes is

$$
\mathbf{B}=\left(\begin{array}{ccccc}
-1 & 0 & 0 & -\frac{1}{2} & -\frac{1}{2} \\
0 & -1 & \frac{1}{2} & -\frac{1}{2} & 0 \\
0 & \frac{1}{2} & -\frac{3}{2} & 0 & 0 \\
-\frac{1}{2} & -\frac{1}{2} & 0 & -\eta-1 & 0 \\
-\frac{1}{2} & 0 & 0 & 0 & -\frac{3}{2}
\end{array}\right) .
$$

As the stability of the CS state should be analyzed according to Eq. (7), the critical pinning strength thus is determined by Eq. (10). Specifically, to make the CS state stable, we should have $\lambda_{1}^{B}<\lambda_{c}=-\sigma_{c} / \varepsilon$. For $\varepsilon=20$ [the parameter used in Fig. 3(a)], we have $\lambda_{c} \approx-0.42$. As $\lambda_{1}^{B}$ is a function of $\eta$, i.e., $\lambda_{1}^{B}=\lambda_{1}^{B}(\eta)$, to find the critical pinning strength $\eta_{c}$, we first need to find the variation of $\lambda_{1}^{B}$ with respect to $\eta$. This is done by numerical simulations and the results are presented in Fig. 5(a1). We see that $\lambda_{1}^{B}(\eta)<\lambda_{c}$ for $\eta>\eta_{c} \approx 0.83$. This prediction is consistent with the numerical results shown in Fig. 3(a2).

According to Eq. (10) and Fig. 5(a1), we can also predict the variation of $\eta_{c}$ with respect to $\varepsilon$. The result is plotted in Fig. 3(a3). We see that the predictions are in good agreement with the numerical results over the scanned region.

As shown in Eq. (12), the stability of the global synchronization state is determined by the largest nonzero eigenvalue of $\mathbf{D}$, namely, $\lambda_{2}^{D}$. To keep the global synchronization state stable, the necessary condition is that $\lambda_{2}^{D}<\lambda_{c}=-\sigma_{c} / \varepsilon$. For 
$\varepsilon=20$, we have $\lambda_{c} \approx-0.28$. By numerical simulations, we plot in Fig. 5(a2) the variation of $\lambda_{2}^{D}$ with respect to $\eta$, which shows that $\lambda_{2}^{D}(\eta)>\lambda_{c}$ in the region of $\eta \in(0,3)$. This explains why global synchronization cannot be reached in Fig. 3(a).

In a similar way, we can also analyze the stability of the CS state when nodes 3 and 9 are pinned [see Fig. 3(b)]. For this case, the enlarged network satisfies reflection symmetry $S_{2}$, which gives the permutation matrix

$$
\mathbf{R}_{S 2}=\left(\begin{array}{ccccccccccc}
1 & 0 & 0 & 0 & 0 & 0 & 0 & 0 & 0 & 0 & 0 \\
0 & 0 & 0 & 0 & 0 & 0 & 0 & 0 & 0 & 1 & 0 \\
0 & 0 & 0 & 0 & 0 & 0 & 0 & 0 & 1 & 0 & 0 \\
0 & 0 & 0 & 0 & 0 & 0 & 0 & 1 & 0 & 0 & 0 \\
0 & 0 & 0 & 0 & 0 & 0 & 1 & 0 & 0 & 0 & 0 \\
0 & 0 & 0 & 0 & 0 & 1 & 0 & 0 & 0 & 0 & 0 \\
0 & 0 & 0 & 0 & 1 & 0 & 0 & 0 & 0 & 0 & 0 \\
0 & 0 & 0 & 1 & 0 & 0 & 0 & 0 & 0 & 0 & 0 \\
0 & 0 & 1 & 0 & 0 & 0 & 0 & 0 & 0 & 0 & 0 \\
0 & 1 & 0 & 0 & 0 & 0 & 0 & 0 & 0 & 0 & 0 \\
0 & 0 & 0 & 0 & 0 & 0 & 0 & 0 & 0 & 0 & 1
\end{array}\right) .
$$

Transforming the coupling matrix into the space spanned by the eigenvectors of $\mathbf{R}_{S 2}$, we have the blocked matrix of the form shown in Eq. (5), with

$$
\mathbf{D}=\left(\begin{array}{ccccccc}
-\eta-1 & 0 & \frac{1}{2} & 0 & \frac{1}{2} & 0 & \sqrt{2} \eta \\
0 & -1 & 0 & 0 & 0 & \frac{\sqrt{2}}{2} & 0 \\
\frac{1}{2} & 0 & -1 & 0 & 0 & \frac{1}{2} & 0 \\
0 & 0 & 0 & -1 & \frac{\sqrt{2}}{2} & 0 & 0 \\
\frac{1}{2} & 0 & 0 & \frac{\sqrt{2}}{2} & -1 & 0 & 0 \\
0 & \frac{\sqrt{2}}{2} & \frac{1}{2} & 0 & 0 & -1 & 0 \\
0 & 0 & 0 & 0 & 0 & 0 & 0
\end{array}\right)
$$

and

$$
\mathbf{B}=\left(\begin{array}{cccc}
-\eta-1 & 0 & -\frac{1}{2} & -\frac{1}{2} \\
0 & -1 & -\frac{1}{2} & 0 \\
-\frac{1}{2} & -\frac{1}{2} & -1 & 0 \\
-\frac{1}{2} & 0 & 0 & -1
\end{array}\right)
$$

As the clusters are intertwined, the stability of the CS state should be evaluated by Eq. (10), which suggests that to make the desired CS state stable, the necessary condition is $\lambda_{1}^{B}(\eta)<$ $\lambda_{c}=-\sigma_{c} / \varepsilon$. For $\varepsilon=25$ [the parameter used in Fig. 3(b)], we have $\lambda_{c} \approx-0.33$. By numerical simulations, we plot in Fig. 5(b1) the variation of $\lambda_{1}^{B}$ with respect to $\eta$. We see that $\lambda_{1}^{B}<\lambda_{c}$ for $\eta>\eta_{c} \approx 0.56$. This prediction agrees very well with the numerical result shown in Fig. 3(b2). The variation of $\lambda_{2}^{D}$ with respect to $\eta$ is plotted in Fig. 5(b2). We see that $\lambda_{2}^{D}>\lambda_{c}$ for $\eta \in(0,3)$, indicating that global synchronization cannot be reached in this region. This explains the numerical results shown in Fig. 3(b). Again, according to Eq. (10) and by the results shown in Fig. 5(b1), we can predict the variation of $\eta_{c}$ with respect to $\varepsilon$, which is plotted in Fig. 3(b3). We see that the predictions agree well with the numerical results.
We finally analyze the stability of the CS state shown in Fig. 4, i.e., pinnings $m=5$ equally spaced nodes in the right-structure network. As the enlarged network satisfies the discrete rotational symmetry $S_{3}$, the $N=11$ nodes are divided into two symmetric groups, $V_{1}=\{1,3,5,7,9\}$ and $V_{2}=\{2,4,6,8,10\}$, and an isolated node (the controller), $V_{3}=\{11\}$. For this case, we have the permutation matrix

$$
\mathbf{R}_{S 3}=\left(\begin{array}{ccccccccccc}
0 & 0 & 1 & 0 & 1 & 0 & 1 & 0 & 1 & 0 & 0 \\
0 & 0 & 0 & 1 & 0 & 1 & 0 & 1 & 0 & 1 & 0 \\
1 & 0 & 0 & 0 & 1 & 0 & 1 & 0 & 1 & 0 & 0 \\
0 & 1 & 0 & 0 & 0 & 1 & 0 & 1 & 0 & 1 & 0 \\
1 & 0 & 1 & 0 & 0 & 0 & 1 & 0 & 1 & 0 & 0 \\
0 & 1 & 0 & 1 & 0 & 0 & 0 & 1 & 0 & 1 & 0 \\
1 & 0 & 1 & 0 & 1 & 0 & 0 & 0 & 1 & 0 & 0 \\
0 & 1 & 0 & 1 & 0 & 1 & 0 & 0 & 0 & 1 & 0 \\
1 & 0 & 1 & 0 & 1 & 0 & 1 & 0 & 0 & 0 & 0 \\
0 & 1 & 0 & 1 & 0 & 1 & 0 & 1 & 0 & 0 & 0 \\
0 & 0 & 0 & 0 & 0 & 0 & 0 & 0 & 0 & 0 & 1
\end{array}\right) .
$$

Transforming the coupling matrix into the space spanned by the eigenvectors of $\mathbf{R}_{S 3}$, we have the blocked matrix of the form shown in Eq. (5), with

$$
\mathbf{D}=\left(\begin{array}{ccc}
0 & 0 & 0 \\
0 & -1 & 1 \\
\sqrt{5} \eta & 1 & -\eta-1
\end{array}\right)
$$

Unlike other cases, here the submatrix B cannot be explicitly given. As the two clusters are intertwined, the stability of the CS state should be analyzed according to Eq. (10) too. For $\varepsilon=$ 24 [the parameter used in Fig. 4(b)], we have $\lambda_{c}=-\sigma_{c} / \varepsilon \approx$ -0.35 . Hence, to make the two clusters synchronizable, the necessary condition is $\lambda_{1}^{B}<\lambda_{c}$. By numerical simulations, we plot in Fig. 5(c1) the variation of $\lambda_{1}^{B}$ with respect to $\eta$, which shows that $\lambda_{1}^{B}<\lambda_{c}$ for $\eta_{c}>0.34$. This prediction agrees with the numerical result shown in Fig. 4(b) (numerically we have $\left.\eta_{c} \approx 0.35\right)$.

Figure 5(c2) plots the variation of $\lambda_{2}^{D}$ with respect to $\eta$. We see that $\lambda_{2}^{D}<\lambda_{c}$ for $\eta>\eta_{g}=0.87$. That is, the enlarged network reaches the global synchronization state for $\eta>\eta_{g}$. This prediction is consistent with the numerical result shown in Fig. 4(b), where it is found that the network is globally synchronized at the critical pinning strength $\eta_{g} \approx 0.85$.

Comparing the results of globally connected and ringstructure networks, we see that the different behaviors that respond to the pinnings, e.g., the relation between $\eta_{c}$ and $m$, are rooted in the different network symmetries created by the pinnings. Specifically, in globally connected network, the network nodes are always divided into two symmetric clusters, whereas in ring-structure network, the configuration of the symmetric clusters varies with $m$ and the pinning locations. This difference leads to the different behavior of $\lambda_{1}^{B}$, which in turn affects the value of $\eta_{c}$. It is worth noting that unlike a globally connected network, in ring-structure network we are not able to obtain analytically the dependence of $\eta_{c}$ on either $N$ or $m$. To find $\lambda_{1}^{B}$, we have to rely on simulations. 

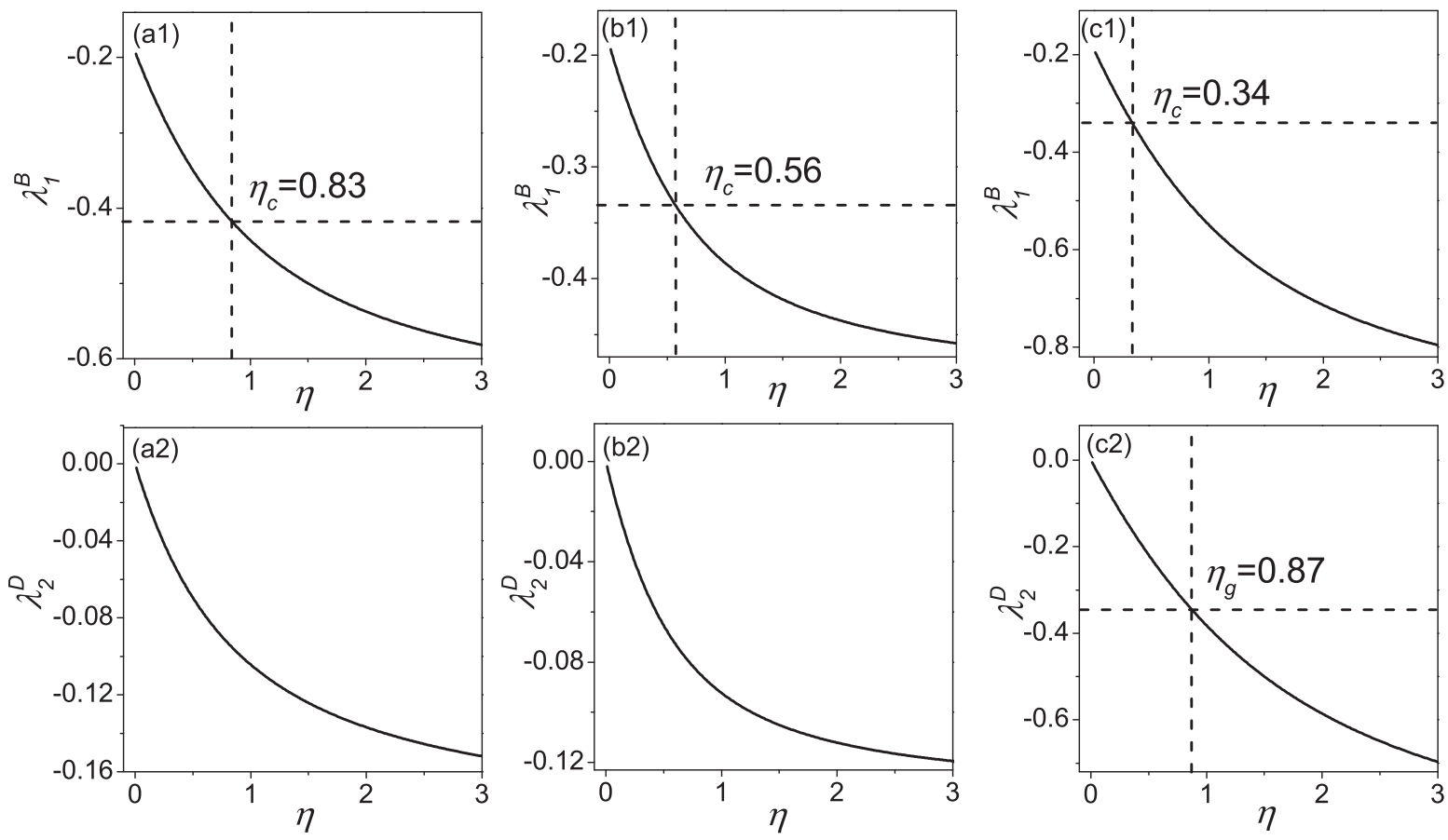

FIG. 5. For the ring-structure network, the variations of $\lambda_{1}^{B}$ (upper row) and $\lambda_{2}^{D}$ (lower row) with respect to $\eta$. (a1),(a2) Pinnings are added on nodes 3 and 8 [see Fig. 3(a1)]. (b1),(b2) Pinnings are added on nodes 3 and 9 [see Fig. 3(b1)]. (c1),(c2) Pinnings are added on nodes 1, 3 , 5, 7, and 9 [see Fig. 4(a)]. The dashed horizontal lines in (a1), (b1), and (c1) denote the critical eigenvalue for cluster synchronization. The dashed horizontal line in (c2) denotes the critical eigenvalue for global synchronization.

\section{DISCUSSIONS AND CONCLUSION}

We would like to point out the difference between the current study and the previous ones on the pinning control of synchronization in complex networks. First, different from previous studies in which the mission of the control is to synchronize all nodes in the network to an external controller (i.e., reaching the state of global synchronization) $[35,37,38]$, the present work concentrates on how to control a network to different CS states of spatial structures. For this reason, the controllability of a network is dependent on not only the network structure and the fraction of pinning nodes, but also the specific CS state to be controlled to, as depicted in Figs. 3 and 4. Second, the present work is also different from the recent studies on pinning control of CS in complex networks $[25,26]$. In pinning CS in complex networks, every node in the network receives a pinning signal, with nodes within the same cluster receive pinnings from the same controller in a small-size control network. As such, the network is globally pinned. Different from this, in our present work pinnings are added onto only a fraction of the nodes in the network. That is, the network is partially pinned. Third, in controlling CS in complex networks, the target states are restricted by the network symmetries. This seriously limits the number of CS states that are able to be controlled to. In contrast, regular networks possess high-order symmetries which, in the presence of pinnings, could be degenerated into various low-order symmetries, potentially supporting the generation of a variety of CS states. This feature is witnessed in both globally connected network (see Figs. 1 and 2) and ringstructure network (see Figs. 3, 4, and 5), where the same network is controlled to different CS states by varying the pinning locations or the number of pinning nodes. Finally, in pinning ring-structure networks, the symmetric clusters are intertwined with each other. As a result, all clusters are stabilized at the same critical pinning strength. This feature is different from that of complex network, in which the symmetric clusters are normally decoupled from each other, and the clusters are synchronized at different critical pinning strengths.

Special emphasis should be given to the approximation that we have made in obtaining the synchronization criteria described by Eqs. (10)-(12). For the general CS state, the synchronization manifolds of the clusters, $\mathscr{X}_{l}$, are desynchronized from each other and are different from the manifold of the controller, $\mathscr{X}_{T}$. As such, the stability of the CS state, in principle, should be evaluated by solving Eqs. (3) and (7) together. This approach, while applicable to the general situation, requires a large amount of computational effort. By the approximation, i.e., the statistical properties of $D \mathbf{F}\left(\mathscr{X}_{l}\right)$ are identical for all cluster synchronization manifolds and can be approximated by $D \mathbf{F}\left(\mathscr{X}_{T}\right)$, the stability of the CS state is determined by Eq. (9). Now, to evaluate whether the CS state is stable, we need only to calculate the variation of $\Lambda$ with respect to $\sigma$ (i.e., the MSF curve), together with the eigenvalues of the transverse matrices $\mathbf{B}^{l}$. This approach is similar to, but different from, the conventional method of MSF in that here the variational equations are transformed first into the eigenspace of the permutation matrix and then into the mode space of the transverse submatrix, while in the standard MSF method the variational equations are directly transformed into the eigenspace of the coupling matrix. Our numerical results shows that at least for coupled chaotic Lorenz oscillators, this approximation is reasonable. As a 
matter of fact, a small mismatch does exist between the theoretical predictions and the numerical results. For instance, we have $\eta_{c} \approx 0.5$ in Fig. 1(b), while theoretical prediction gives $\eta_{c} \approx 0.55$.

To conclude, we have studied numerically and theoretically the pinning control of CS in regular networks, and found that depending on the number of pinning nodes and the pinning locations, the same network can be controlled to different CS states. The stability criteria of CS states in different scenarios have been given explicitly, and the theoretical predictions are in good agreement with the numerical results. Our study shows the flexibility of pinning control in generating CS in regular networks, and highlights, from a different perspective, the importance of network symmetry on network collective behaviors.

\section{ACKNOWLEDGMENT}

This work was supported by the National Natural Science Foundation of China under Grant No. 11875182.
[1] A. S. Pikovsky, M. G. Rosenblum, and J. Kurths, Synchronization: A Universal Concept in Nonlinear Science (Cambridge University Press, Cambridge, 2001).

[2] D. Hansel, G. Mato, and C. Meunier, Clustering and slow switching in globally coupled phase oscillators, Phys. Rev. E 48, 3470 (1993).

[3] V. N. Belykh and E. Mosekilde, One-dimensional map lattices: Synchronization, bifurcations, and chaotic structures, Phys. Rev. E 54, 3196 (1996).

[4] M. Hasler, Yu. Maistrenko, and O. Popovych, Simple example of partial synchronization of chaotic systems, Phys. Rev. E 58, 6843 (1998).

[5] Y. Zhang, G. Hu, H. A. Cerdeira, S. Chen, T. Braun, and Y. Yao, Partial synchronization and spontaneous spatial ordering in coupled chaotic systems, Phys. Rev. E 63, 026211 (2001).

[6] A. Pikovsky, O. Popovych, and Yu. Maistrenko, Resolving Clusters in Chaotic Ensembles of Globally Coupled Identical Oscillators, Phys. Rev. Lett. 87, 044102 (2001).

[7] I. A. Heisler, T. Braun, Y. Zhang, G. Hu, and H. A. Cerdeira, Experimental investigation of partial synchronzation in coupled chaotic oscillators, Chaos 13, 185 (2003).

[8] C. S. Zhou and J. Kurths, Hierarchical synchronization in complex networks with heterogeneous degrees, Chaos 16, 015104 (2006).

[9] J. Zhang, Y. Yu, and X. G. Wang, Synchronization of coupled metronomes on two layers, Front. Phys. 12, 120508 (2017).

[10] M. M. Norton, N. Tompkins, B. Blanc, M. C. Cambria, J. Held, and S. Fraden, Dynamics of Reaction-Diffusion Oscillators in Star and Other Networks with Cyclic Symmetries Exhibiting Multiple Clusters, Phys. Rev. Lett. 123, 148301 (2019).

[11] M. Golubitsky, I. Stewart, and D. G. Schaeffer, Singularities and Groups in Bifurcation Theory (Springer-Verlag, Berlin, 1985).

[12] J. F. Heagy, L. M. Pecora, and T. L. Carroll, Short Wavelength Bifurcations and Size Instabilities in Coupled Oscillator Systems, Phys. Rev. Lett. 74, 4185 (1995).

[13] L. M. Pecora, Synchronization conditions and desynchronizing patterns in coupled limit-cycle and chaotic systems, Phys. Rev. E 58, 347 (1998).

[14] L. M. Pecora and T. L. Carroll, Master Stability Functions for Synchronized Coupled Systems, Phys. Rev. Lett. 80, 2109 (1998).

[15] G. Hu, J. Z. Yang, and W. Liu, Instability and controllability of linearly coupled oscillators: Eigenvalue analysis, Phys. Rev. E 58, 4440 (1998).
[16] L. Huang, Q. Chen, Y.-C. Lai, and L. M. Pecora, Generic behavior of master-stability functions in coupled nonlinear dynamical systems, Phys. Rev. E 80, 036204 (2009).

[17] D. J. Watts and S. H. Strogatz, Collective dynamics of "smallworld" networks, Nature (London) 393, 440 (1998).

[18] A.-L. Barabási and R. Albert, Emergence of scaling in random networks, Science 286, 509 (1999).

[19] B. Ao and Z. G. Zheng, Partial synchronization on complex networks, Europhys. Lett. 74, 229 (2006).

[20] G. Russo and J. J. E. Slotine, Symmetries, stability, and control in nonlinear systems and networks, Phys. Rev. E 84, 041929 (2011).

[21] C. Fu, Z. Deng, L. Huang, and X. G. Wang, Topological control of synchronous patterns in systems of networked chaotic oscillators, Phys. Rev. E 87, 032909 (2013).

[22] C. Fu, W. Lin, L. Huang, and X. G. Wang, Synchronization transition in networked chaotic oscillators: The viewpoint from partial synchronization, Phys. Rev. E 89, 052908 (2014).

[23] L. M. Pecora, F. Sorrentino, A. M. Hagerstrom, T. E. Murphy, and R. Roy, Cluster synchronization and isolated desynchronization in complex networks with symmetries, Nat. Commun. 5, 4079 (2014).

[24] M. Golubitsky and I. Stewart, Recent advances in symmetric and network dynamics, Chaos 25, 097612 (2015).

[25] W. Lin, H. Fan, Y. Wang, H. Ying, and X. G. Wang, Controlling synchronous patterns in complex networks, Phys. Rev. E 93, 042209 (2016).

[26] W. Lin, H. Li, H. Ying, and X. G. Wang, Inducing isolateddesynchronization states in complex network of coupled chaotic oscillators, Phys. Rev. E 94, 062303 (2016).

[27] F. Sorrentino, L. M. Pecora, A. M. Hagerstrom, T. E. Murphy, and R. Roy, Complete characterization of the stability of cluster synchronization in complex dynamical networks, Sci. Adv. 2, e1501737 (2016)

[28] T. Nishikawa and A. E. Motter, Network-complement transitions, symmetries, and cluster synchronization, Chaos $\mathbf{2 6}$ 094818 (2016).

[29] M. T. Schaub, N. O’Clery, Y. N. Billeh, J.-C. Delvenne, R. Lambiotte, and M. Barahona, Graph partitions and cluster synchronization in networks of oscillators, Chaos 26, 094821 (2016).

[30] A. B. Siddique, L. M. Pecora, J. D. Hart, and F. Sorrentino, Symmetry- and input-cluster synchronization in networks, Phys. Rev. E 97, 042217 (2018). 
[31] B. Cao, Y. F. Wang, L. Wang, Y. Z. Yu, and X. G. Wang, Cluster synchronization in complex network of coupled chaotic circuits: An experimental study, Front. Phys. 13, 130505 (2018).

[32] J. D. Hart, Y. Zang, R. Roy, and A. E. Motter, Topological Control of Synchronization Patterns: Trading Symmetry for Stability, Phys. Rev. Lett. 122, 058301 (2019).

[33] W. Wu, W. Zhou, and T. Chen, Cluster synchronization of linearly coupled complex networks under pinning control, IEEE Trans. Circuits Syst. I 56, 829 (2009).

[34] W. Lu, B. Liu, and T. Chen, Cluster synchronization in networks of coupled nonidentical dynamical systems, Chaos 20, 013120 (2010).

[35] X. F. Wang and G. R. Chen, Pinning control of scale-free dynamical networks, Physica A 310, 521 (2002).
[36] X. Li, X. F. Wang, and G. Chen, Pinning a complex dynamical network to its equilibrium, IEEE Trans. Circuits Syst. I 51, 2074 (2004).

[37] F. Sorrentino, M. di Bernardo, F. Garofalo, and G. R. Chen, Controllability of complex networks via pinning, Phys. Rev. E 75, 046103 (2007).

[38] L. Yang, X. G. Wang, Y. Li, and Z. M. Sheng, On the pinning strategy of complex networks, Europhys. Lett. 92, 48002 (2010).

[39] Y. Kuramoto and D. Battogtokh, Coexistence of coherence and incoherence in nonlocally coupled phase oscillators, Nonlinear Phenom. Complex Syst. 5, 380 (2002).

[40] D. Abrams and S. Strogatz, Chimera states for Coupled Oscillators, Phys. Rev. Lett. 93, 174102 (2004).

[41] Y. Wang, L. Wang, H. Fan, and X. G. Wang, Cluster synchronization in networked nonidentical chaotic oscillators, Chaos 29, 093118 (2019). 